\title{
Acquired neuromuscular weakness In Intensive Care Unit: Review article
}

\author{
Mohamed Hamdy Elghotmy (M.Sc.) *, Hamdy Elewa (MD)**, Mohamed Rabea (MD)** \\ *Anaesthesia, Intensive care \& and Pain Management Department, Ministry of Health, Menoufia, Egypt \\ **Anaesthesia, Intensive care \& and Pain Management Department, Faculty of Medicine, Benha University, \\ Benha, Egypt \\ Corresponding Author: \\ Mohamed Hamdy Elghotmy, M.Sc. \\ Shebin El-Kom City, Menoufia governorate, Egypt
}

\begin{abstract}
A substantial number of patients admitted to the ICU because of an acute illness, complicated surgery, severe trauma, or burn injury will develop a de novo form of muscle weakness during the ICU stay that is referred to as "intensive care unit acquired weakness" (ICUAW). This ICUAW evoked by critical illness can be due to axonal neuropathy, primary myopathy, or both. Underlying pathophysiological mechanisms comprise microvascular, electrical, metabolic, and bioenergetic alterations, interacting in a complex way and culminating in loss of muscle strength and/or muscle atrophy. ICUAW is typically symmetrical and affects predominantly proximal limb muscles and respiratory muscles, whereas facial and ocular muscles are often spared. ICUAW is diagnosed in awake and cooperative patients by bedside manual testing of muscle strength and the severity is scored by the Medical Research Council sum score. In cases of atypical clinical presentation or evolution, additional electrophysiological testing may be required for differential diagnosis. The cornerstones of prevention are aggressive treatment of sepsis, early mobilization, preventing hyperglycemia with insulin, and avoiding the use parenteral nutrition during the first week of critical illness. Future research should focus on new preventive and/or therapeutic strategies for this detrimental complication of critical illness and on clarifying how ICUAW contributes to poor longer-term prognosis.
\end{abstract}

Keywords: Acquired neuromuscular weakness, future directions, ICU, mortality, treatment

\section{Introduction}

Acquired weakness during hospitalization for critical illness is increasingly recognized as a common and important clinical problem. Weakness acquired in intensive care unit ICU and related acquired neuromuscular dysfunctions occur in large percentage of critically ill patients. Neuromuscular abnormalities culminating in skeletal muscle weakness occur very commonly in critically ill patient. Critically ill patients may suffer from acute neuropathy or myopathy causing muscle weakness and paralysis.[1] The terminology regarding these neuromuscular alterations is confusing and terms such as critical illness polyneuropathy, critical illness myopathy, intensive care unit weakness or paralysis are often used interchangeably.[2] ICU acquired neuromuscular abnormalities are typically divided into two discrete classes: polyneuropathy and myopathy. However, it is likely that these two entities commonly coexist, with myopathy being the most common cause of weakness.[3] Major risk factors for ICU acquired neuromuscular abnormalities include sepsis, corticosteroids administration, hyperglycemia, with other associated factors including neuromuscular blockade and increasing severity of illness. The pathogenesis of these disorders is not well defined, but probably involves inflammatory injury of nerve and / or muscle that is potentiated by functional denervation and corticosteroid.[4] Electrolyte disorders frequently develop in critically ill patients during course of stay in the ICU. Therefore, ICU patients are routinely monitored for electrolyte disorders. ICU acquired neuromuscular abnormalities are associated with multiple adverse outcomes, including higher mortality, prolonged duration of mechanical ventilation, and increased length of stay.[5] Electrophysiological investigations of peripheral nerves and muscles are important to define the nature of the pathological process, achieve a differential diagnosis and predict long-term prognosis. Measurement of muscle strength using Medical Research Council score or handgrip dynamometry is important to define the severity of muscle strength impairment and to predict short-term morbidity and hospital mortality. Early physical rehabilitation and occupational therapy associated with interruption of sedation may result in better outcomes.[6] This review aims to update the available knowledge regarding define ICU-acquired neuromuscular abnormalities and identify risk factors, causes and management to decrease multiple adverse outcomes.

\section{Definition and classification}

It would appear that virtually every author who has published on this subject had decided to create their own name for the same cluster of clinical findings. is an excellent discussion of this lawless swamp. They discovered eighteen definitions just in the 1990-2009 timeframe ("intensive care unit-acquired neuromyopathy", "ICU-acquired paresis", "polyneuropathy in critically ill patients", "acute necrotising myopathy of intensive care", and so on). Stevens et al proposed their own definition to bring some order into this environment:

"ICU-acquired weakness ... is clinically detected weakness in critically ill patients in whom there is no plausible etiology other than critical illness." [7] 
Within this clinical definition, patients who actually do undergo formal EMG or nerve conduction testing may be further subclassified as CIP, CIM, or CINM. How weak does one have to be, and what defines "ICUacquired"? Well. Manual muscle testing using the Medical Research Council (MRC) score is probably the best-known technique. [8] explore in detail; for this short summary it will suffice to say that it requires testing each of the muscle groups of the extremities and generating a six-point ordinal score. This scoring system is usually referred to as the MRC-SS (SS stands for "summed score"), and was developed by Kleyweg to track the recovery of patients with Guillain-Barré syndrome.[9] To simplify revision, the testing scale is reproduced here:

\begin{tabular}{|c|l|}
\hline Grade & Manual Muscle Test \\
\hline $\mathbf{5}$ & Movement against gravity plus full resistance \\
\hline $\mathbf{4}$ & Movement against gravity plus some resistance \\
\hline $\mathbf{3}$ & $\begin{array}{l}\text { Completes the available test range of motion } \\
\text { against gravity, but tolerates no resistance }\end{array}$ \\
\hline $\mathbf{2}$ & $\begin{array}{l}\text { the patient completes full or partial range of } \\
\text { motion with gravity eliminated }\end{array}$ \\
\hline $\mathbf{1}$ & Slight contractility without any movement \\
\hline $\mathbf{0}$ & No evidence of contractility (complete paralysis) \\
\hline
\end{tabular}

Six muscle groups are tested bilaterally (shoulder abduction, elbow extension, wrist extension, hip flexion, knee extension, ankle dorsiflexion). The total score can therefore range from 0 (complete paralysis) to 60 (fully preserved muscle power).

The real incidence of ICU-AW is unclear and varies between studies. This variability reflects different populations, risk factors, definitions of this syndrome, absence of diagnostic criteria widely validated and the difficulty to differentiate all clinical forms adequately. It ranges between $7 \%$ in patients undergoing liver transplantation to $33 \%$ in patients admitted to the intensive care unit with status asthmaticus.[10] Recent reviews report an incidence of 30 to $46 \%$, mainly, secondary to sepsis and multi-organ dysfunction syndrome (MODS). The incidence is also higher (30-60\%) in patients with acute respiratory distress syndrome (ARDS), especially in patients with longterm (> 1 week) duration of mechanical ventilation. Seventy percent of patients with sepsis may suffer from CIP. In children, the risk is less well defined as there are no prospective clinical and electrophysiologic studies.[11]

\section{Etiology}

The causes of weakness in the ICU are multi-factorial and causative factors include immobility, preexisting weakness, underlying medical conditions, and ICU associated weakness (ICUAW). The underlying mechanism for neuromuscular dysfunction in ICUAW includes a combination of axonal degeneration, muscle breakdown, and sodium channel dysfunction with ensuing muscle membrane hypoexcitability. [12] The causes of delirium are also multifactorial including current medical conditions, past medical history and chronic diseases, premorbid cognitive function, acute pain, medications, infection, and the ICU environment itself.[13]

\section{Epidemiology}

Epidemiology of ICU-Acquired Weakness Neuromuscular abnormalities long have been described in connection with critical illness. In 1892, Sir William Osler reported "rapid loss of flesh" in patients with prolonged sepsis. Mertens described polyneuropathy in patients in a coma in the 1960s; in 1977, evidence of acute myopathy was reported in a patient with status asthmaticus. In 2002, De Jonghe et al reported an incidence of ICU-acquired paresis, as measured by means of a Medical Research Council (MRC)-graded manual muscle strength score $<48$ (consistent with severe weakness reflecting inability to resist gravity) in $25.3 \%$ (24 of 95) of patients receiving mechanical ventilation for $\$ 7$ days who survived to awakening and could follow commands.[14] Electrophysiology (EP) testing performed in all patients with persisting weakness showed reduced compound motor action potentials (CMAPs) and sensory nerve action potentials (SNAPs) with abnormal spontaneous muscle activity consistent with sensorimotor axonal peripheral neuropathy. Subsequent review of muscle biopsy specimens in 10 patients demonstrated type II fiber atrophy with myosinolysis consistent with primary myopathy and neurogenic muscle atrophy.[15]

\section{Pathophysiology}

ICU-acquired weakness (ICUAW) in patients who are critically ill commonly manifests in three ways: polyneuropathy, myopathy, and/or muscle atrophy. 6 Neuromuscular dysfunction is identified using EP testing. Motor (commonly the peroneal) and sensory (commonly the sural) nerves are stimulated (Fig 1) with increasing stimulus to elicit distal muscle depolarization. Abnormalities in the resultant action potential result from either nerve or muscle injury or a combination of both. Critical illness polyneuropathy (CIP) is characterized by a symmetric, distal sensory-motor axonal polyneuropathy affecting limb and respiratory muscles, as well as sensory and autonomic nerves.[4] EP studies demonstrate abnormal sensory and motor responses with a reduction in the CMAP and SNAP amplitudes. Despite abnormal nerve conduction study (NCS) results, histologic analysis of sensory nerves in patients with CIP generally appear normal early in the course of illness, with axonal degeneration evident only late in the course.[16] Direct muscle stimulation with a stimulating needle electrode demonstrates preserved CMAP amplitude differentiating neuropathy from myopathy. Needle electromyography (EMG) may demonstrate shortterm changes associated with axonal loss and reduced recruitment. A number of theories have emerged regarding the cause of the development of CIP, including loss of the blood-nerve barrier, in excitability of the endoneurial membrane, and direct toxic effects from ICU therapies including hyperglycemia or lipids in parenteral nutrition.[17]

Critical illness myopathy (CIM) is characterized by limb and respiratory muscle weakness with retained sensory function. EP testing demonstrates reduced CMAP amplitudes with preservation of sensory responses. Needle EMG may demonstrate short-term changes and, if the patient is able to participate, myopathic motor unit action potentials. Direct muscle stimulation may demonstrate low-amplitude CMAP from both muscle and nerve.[5] Histologic study of muscle biopsy specimens demonstrates atrophy with preferential loss of thick filaments reflecting myosin loss and muscle necrosis. Proposed causes for the muscle destruction seen in 
CIM include chemokine-induced autophagy of muscle, muscle membrane in excitability, acquisition of channelopathies, or direct toxic effects of ICU care including corticosteroids or neuromuscular blockade (NMB).[18]

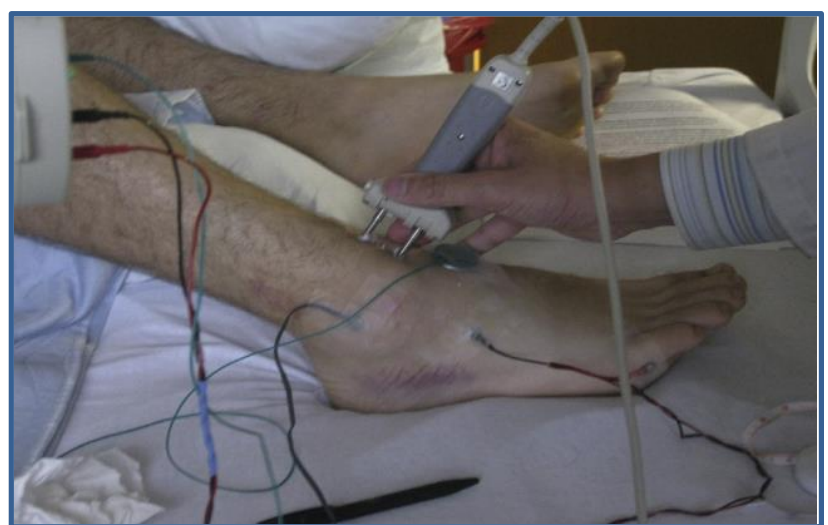

Figure 1: Single fibular nerve conduction study. In a patient who is supine and normothermic, the recording active electrode (black) is placed over the extensor digitorum brevis muscle. The reference electrode (red) is placed over the fifth metatarsal phalangeal joint. Stimulation occurs 8 $\mathrm{cm}$ proximal to the active electrode, slightly lateral to the tibialis anterior tendon. Ground electrode (green) is placed between the stimulation site and the recording active electrode.

\section{Pathophysiological mechanisms}

During the evolution of critical illness different mechanisms of injury trigger and feedback to develop an adverse microenvironment for muscles and nerves. The muscular and nervous affectation does not escape the systemic involvement of different organs as a result of massive release of cytokines and other mediators that activate different cascade of mediators which produce electrical, metabolic, microvascular and bioenergetic disturbances (Figure 2) [19].

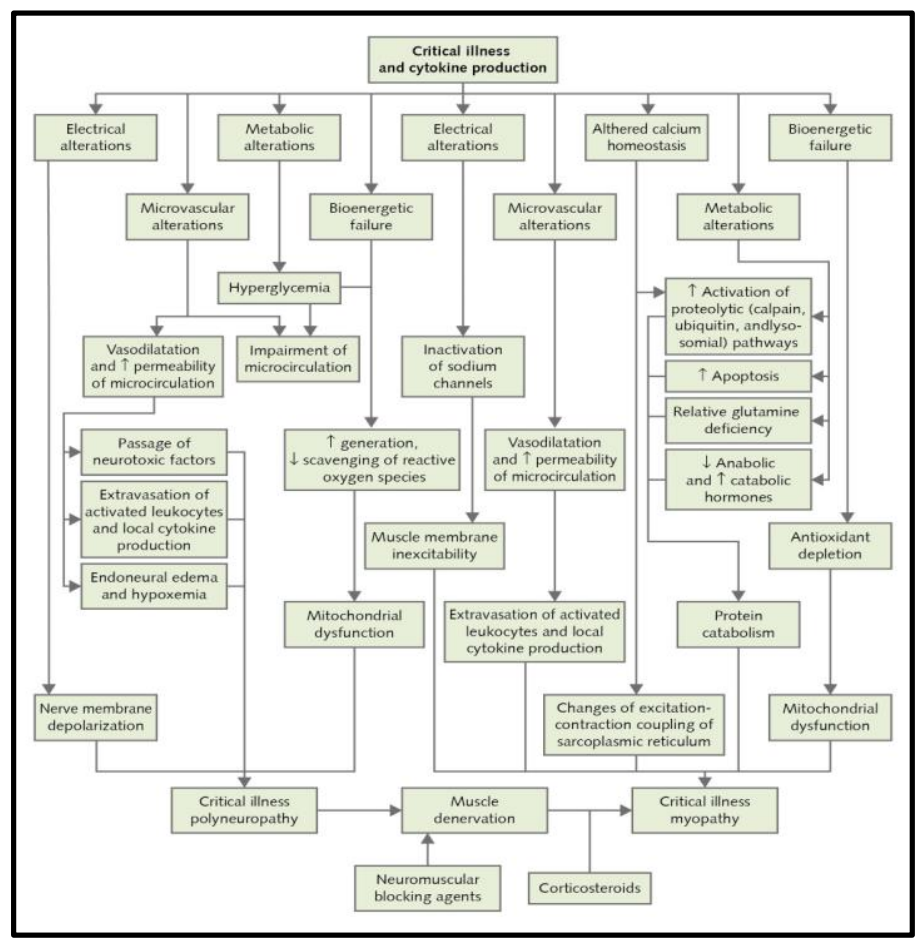

Figure 2. Pathophysiological mechanisms of ICU-AW

\section{Risk factors}

Undoubtedly, important risk factors are age, sepsis, the duration and the severity of illness, as measured by ICU admission Acute Physiology and Chronic Health Evaluation score or Sequential Organ Failure Assessment score, systemic inflammatory response syndrome (SIRS), and MODS [20]. The use of neuromuscular blocking agents, vasopressors, corticosteroids, aminoglycosides and opioids, principally fentanyl, were also associated to ICU-AW. Only anecdotal reports link ICU-AW and administration of parenteral nutrition with the speculation that the use of intravenous fat emulsions with high amounts of polyunsaturated fatty acids could cause axonopathy [7]. Furthermore, additional risk factors include neurological compromise, hyperglycemia, hypoalbuminemia, hyperosmolarity, parenteral nutrition, renal failure with or without replacement therapy, female sex, joint contractures, and muscle wasting from catabolism and physical inactivity also contribute to ICU-AW. Short-term immobility impairs microvascular function and induces insulin resistance [21], increases production of pro-inflammatory cytokines and reactive oxygen species, resulting in muscle proteolysis and a net loss of muscle protein and subsequent muscle weakness. Prolonged immobility leads to decreased muscle protein synthesis, increased muscle catabolism, and decreased muscle mass, especially in the lower extremities. However, the presence of so many risk factors suggests that several factors act synergistically in the development of ICU-AW.[20]

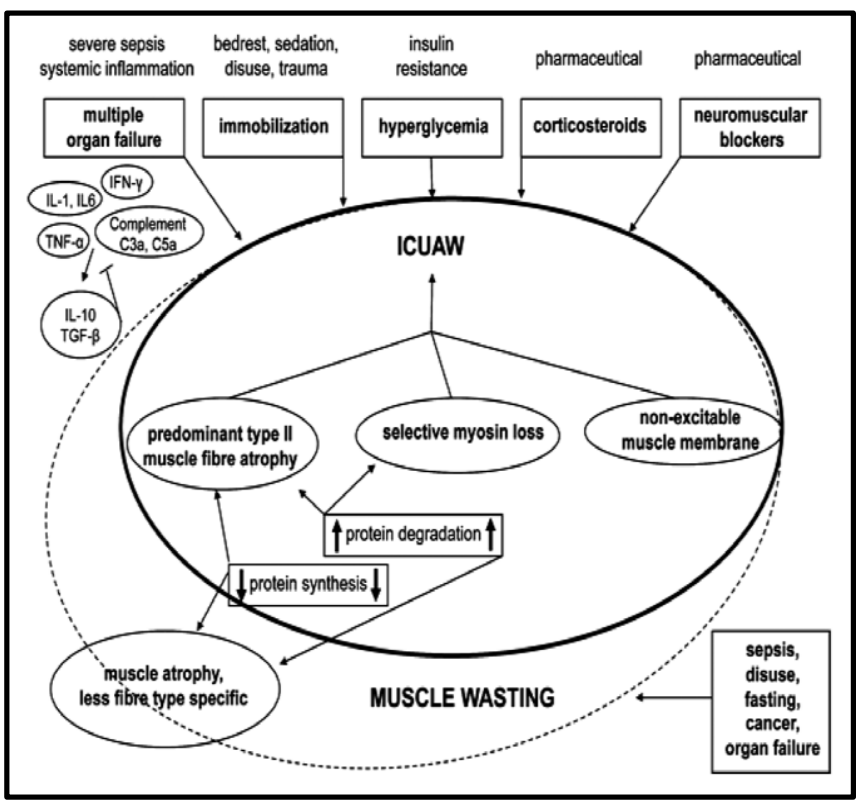

Figure 3: Risk factors involved in muscle wasting and ICU-acquired weakness (ICU-AW) [18]. IFN, interferon; IL, interleukin; TGF, transforming growth factor; TNF, tumor necrosis factor.

Clinical Picture: evaluation and diagnostic criteria for ICU-AW

The classical clinical findings are flaccid tetra paresis, frequently symmetrical with hypo or areflexia (major finding); atrophy and loss of muscle mass (minor finding). An initial examination should be performed upon admission to the ICU, with subsequent examinations paired with daily awakenings or when patients show signs of clinical 
improvement, allowing cooperation with a more comprehensive examination. The diagnosis of ICU-AW should begin with routine physical and neurological examination of a patient that is weak following an episode of critical illness with the use of the Medical Research Council (MRC) scale.[22] The MRC score is a tool that help to graduate the muscle strength of various muscle groups in the upper and lower extremities. It ranges from 0 to 5 , with higher scores indicating greater muscle strength. It has maximal punctuation of 60 . A combined score of 48 points is a cut-off for presumptive diagnosis of ICU-AW. Patients with ICU-AW according to the MRC score should undergo serial evaluations, and if persistent deficits are noted, electrophysiological studies, muscle biopsy, or both are warranted. Unfortunately, the MRC scale has several limitations due to discrimination and a potential ceiling effect, and we need better bedside tools for identifying the presence of ICU-AW.[23]

Three major features usually point toward CNS involvement: asymmetric neurologic signs, altered mental status, and cranial nerve palsies (brain stem lesion). Appropriate imaging and electroencephalogram usually provide the diagnosis. Many spinal cord lesions, such as acute transverse myelitis, epidural abscess, and spinal cord infarct, may present as causes of ICU-AW.[1] In the presence of flaccid weakness due to spinal shock, upper versus lower motor neuron paralysis cannot be distinguished. Presence of sensory level on trunk (when it is possible to investigate), Babinski sign, flexor spasms, loss of anal reflex, loss of sphincter control, and arms weaker than legs are some useful signs of spinal cord involvement. Magnetic resonance imaging of the spine is the most useful procedure in any suspicion of a spinal cord lesion. The main clinical features of neuromuscular diseases are weakness and wasting of extremities, hypotonia, and hyporeflexia/areflexia, with or without respiratory and/or cranial musculature involvement.[24]

Some helpful clinical signs are asymmetric weakness and fasciculations (ALS, viral poliomyelitis); paresthesia, sensory deficits, and distal symmetric weakness (peripheral neuropathy); cranial nerve palsies and dysautonomia (GBS); and combination of ptosis and weakness of eye closure (myasthenia gravis, prolonged neuromuscular junction blockade). Given the complexities of ICU-AW, a standard and practical definition is evidently difficult.[25]

\section{Proposed diagnostic algorithm}

To optimize the likelihood of patient interaction for neuromuscular assessments, we advocate the implementation of sedation protocols. Daily interruption of sedative infusions confers the opportunity for serial neuromuscular examination and reduces the duration of mechanical ventilation. Careful implementation of the structured MRC examination should be employed and documented serially as a matter of routine. Patients exhibiting fixed or focal motor defects or persistent altered sensorium despite adequate sedation washout should undergo more advanced diagnostics (i.e., CNS imaging, electrophysiologic studies, and/or muscle biopsy), (Figure 4).[26]

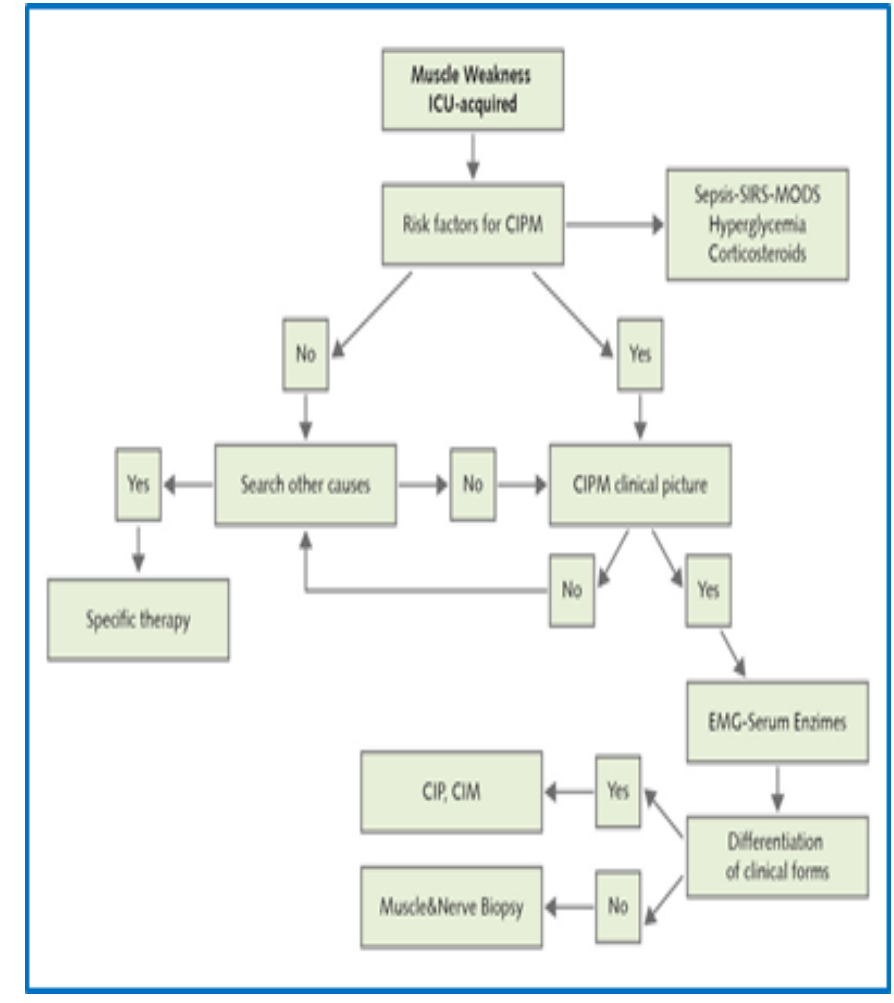

Figure 4. Practical algorithm for diagnosis of critical illness polyneuromyopathy.

The decision to perform electrophysiologic testing and/or muscle biopsy in routine care has created an ongoing debate in the medical literature about its utility. Advocates for routine clinical examination who reserve neurophysiological testing and biopsy for unusual or severe instances of weakness cite the limitations, costs, and risks of this testing is a useful and efficient strategy. Establishing a highly specific or physiologically based diagnosis does not translate to a specific pharmacologic therapy.[27]

\section{Differential diagnoses}

\section{Clinical evaluation of muscle strength}

Current guidelines recommend a clinical diagnosis of ICUAW, made by bedside evaluation of the muscle strength with the use of the Medical Research Council (MRC) sum score.[1] This score appoints a value between 0 (no contraction at all) and 5 (normal muscle strength) for each of 12 muscle groups, including shoulder abduction, elbow flexion, wrist extension, hip flexion, knee extension, and dorsiflexion of the ankle, all scored bilaterally (Table 1).[28] The sum score ranges between 0 and 60 , and ICUAW is diagnosed with a total $<48$. Additional electrophysiological examination for clinical purposes is only indicated when the clinical presentation or evolution is atypical or when focal deficit is present. The MRC sum score has a number of limitations.[29] It does not detect the cause of weakness and cannot differentiate between CIP and CIM. Also, patients need to be awake and fully cooperative; a subset of patients, ranging between $10 \%$ and $75 \%$, do not fulfill this criterion during their ICU stay. Reproducibility in critically ill patients was reported to be good, but this was not confirmed by other studies.[30]

\section{Electrophysiological testing}


Because of the shortcomings of the MRC sum score, the interest in alternative diagnostic tools remains. Full electrophysiological testing, including nerve conduction studies (NCS) and electromyography (EMG), is cumbersome, not readily available in many ICUs, time consuming, and costly. NCS typically reveal reduced compound muscle action potentials (CMAPs); in the case of (coexistent) CIP, sensory nerve action potentials (SNAPs) may be reduced, and nerve conduction velocity is normal or only slightly reduced.[31] In both CIP and CIM, spontaneous electrical activity may be present on EMG. SNAPs in critically ill patients may also be reduced due to edema. Voluntary muscle contraction, which requires a cooperative patient, or alternatively more complex electrophysiological methods such as direct muscle stimulation can be used to differentiate between CIP and CIM. Simplified electrophysiological screening using the peroneal nerve CMAP, potentially supplemented by the sural nerve amplitude, may be used to identify patients who require more extensive testing [32].

\section{Biomarkers}

The use of biomarkers could allow better targeting of future novel therapies. Creatine kinase may be increased in patients with ICUAW but is not a good biomarker. Plasma levels of neurofilaments, which are biomarkers of axonal injury, are also elevated in patients with ICUAW.[33] Peak neurofilament levels showed good discriminative power for weakness but this peak only occurred after patients were clinically evaluable and therefore did not allow early diagnosis. Although no validated biomarkers are available currently, identification of new mediators involved in the development of ICUAW, such as GDF-15, may represent promising future candidates.[33]

\section{Treatment and management}

In the absence of specific therapy, the management involves the close collaboration of neurologists, neurophysiologists, and intensivists. The therapeutic strategies should be directed to the control of sepsis, SIRS and organ dysfunction and includes antibiotics to counteract infection, surgical drainage of an infected focus, and the use of inotropic drugs and fluid replacement to control hypotension. Several attempts at controlling early stage of the SIRS have shown variable results.[26] These attempts include monoclonal and polyclonal antibodies directed against bacterial endotoxin, monoclonal antibodies to tumor necrosis factor-alpha, fusion protein constructs of soluble tumor necrosis factor receptors, IL-1 receptor antagonists, the platelet activating factor receptor antagonist, BN5202, and the $\mathrm{N}$-acetylcysteine. There also was no beneficial effect of reducing disease severity using high intravenous immunoglobulins, [34] and IgM-enriched intravenous immunoglobulin. Avoiding or minimizing some pharmacological agents, such as corticosteroids and neuromuscular blocking agents represent the best strategy way for early recovering of muscle and nerve.[35]

Finally, over-sedation has economic consequences due to extension of stay in ICU and in the hospital. It is advisable as far as possible to implement protocols of daily interruption of sedation, in order to withdrawal as quickly as possible.[26] De Jonghe et al. noted that use of a sedation algorithm designed to allow patients to be more alert was associated with a 50\% reduction in pressure sores, presumably because of reductions in sedative-related immobilization.[36] Furthermore, the interruption of daily sedative use during mechanical ventilation increases the percentage of days during which patients are awake and able to follow commands. The changes in ICU care to reduce sedative use improve in-hospital activity levels in a group of patients in the medical ICU.[37]

Insulin antagonizes the different routes of damage generated during hyperglycemia, and also has neuroprotective effects, so it is the agent of choice for blood glucose control strategies. Insulin exerts anti-inflammatory effects, endothelial protection, improvement of dyslipidemia, and neuroprotective effects in animals and is also an anabolic hormone.[26] Although intensive glucose control was earlier thought to be helpful in reducing the incidence and severity of critical illness polyneuropathy, the NICE-SUGAR Study argues against tight glucose control given the higher mortality risk associated with increased moderate to severe hypoglycemia.[38] Intensive insulin therapy should be avoided due to the risk of hypoglycemia. The American Diabetes Association currently recommends target blood glucose of 144 to $180 \mathrm{mg}$ per deciliter to reduce the risk of hypoglycemia in critically ill patients.[39] Therefore, according to a 2014 Cochrane update, intensive insulin therapy reduces ICU-AW (risk ratio 0.65), and it reduces the duration of mechanical ventilation, intensive care unit stay, and 180-day mortality. Because hypoglycemia is a risk, Cochrane reviewers recommend that consequences and prevention of hypoglycemia need further study.[40]

The implementation of an early mobility and intensive rehabilitation program improves outcomes in ICU-AW patients. When compared with a case series of patients with ICUAW who did not receive structured physical therapy, evidence suggested those who receive physical rehabilitation were more frequently discharged home rather than to a rehabilitative facility, although confidence intervals included no difference.[41] Passive mobilization prevents muscle atrophy, profound venous thrombosis and decubitus ulcers. Physical and occupational therapies increase the possibility to achieve good functional outcomes. Daily exercises especially targeted to the diaphragm and respiratory muscles help greatly in the process of weaning from mechanical ventilation. Other interventions show promise, but fewer data proving patient benefit exists.[26] Furthermore, execution of a rehabilitation program requires a collaborative effort among members of the multidisciplinary team to coordinate, care for, and provide safe mobilization of patients in intensive care units. Finally, electrical muscle stimulation (EMS) may be an alternative therapy for patients who cannot actively exercise or others disabling comorbidities and in patients unable to cooperate. This technique may maintain muscle strength and joint range of motion, improve outcomes of ventilation, and reduce activity limitations.[42]

ICUAW, Mortality and Long-term Physical Function

An increasing number of studies highlight the burden of impairment seen after critical illness, particularly after ARDS, but few attempts to link ICUAW with subsequent morbidity and mortality. ICUAW is an increasingly recognized independent predictor of mortality in patients who are critically ill. In a propensity-matched cohort of patients who were critically ill, MRC-defined ICUAW was associated with a $30 \%$ lower likelihood of being alive at hospital discharge (hazard ratio, 0.70; 95\% CI, 0.55-0.86; P 
$1 / 4.008)$, with a $13 \%$ increase in mortality at 1 year.[43] This finding supports those of prior observational studies, suggesting increased odds of death associated with ICUAW independent of other ICU factors. Although it seems intuitive that ICUAW would impact long-term physical function after critical illness, few data support this assumption to date.[13] In the Improving Care of Acute Lung Injury Patients study, Fan et al examined the association between ICUAW (defined as an MRC strength score < 80\% maximum) with post discharge strength measured by means of serial testing at 3, 6, 12, and 24 months after ARDS. In the cohort, $36 \%$ of patients exhibited signs of ICUAW at hospital discharge, with the prevalence decreasing to $22 \%$ at 3 months, $15 \%$ at 6 months, $14 \%$ at 12 months, and 9\% at 24 months after ARDS.56 Manual muscle strength increased significantly over time, with an increase in total MRC score from 50 at hospital discharge to 57 at 24 months.[44] Despite improvements in overall strength, physical functioning health-related quality-of-life subscales remained significantly lower than age-adjusted norms at all time points, achieving only $72 \%$ of baseline values at 24 months.56 Patients with documented ICUAW maintained significantly worse handgrip strength and reported worse physical functioning health-related quality of life.[3]

\section{Prognosis}

ICU-AW is associated with increased mortality rate and rehabilitation problems. Rehabilitation therapy is likely to be beneficial in speeding the recovery of these patients. Prognosis for recovery from CIM is more favorable than that of CIP alone, unless muscle necrosis is severe, or CINM.[4] Children fair better than adults for a favorable recovery was thought to be as low as $50 \%$. However, if the polyneuropathy is severe, recovery may take months. In especially severe cases, recovery may not occur. Neurophysiological studies performed at intervals can aid in determining prognosis of recovery.[46]

\section{Conclusions and Future directions}

ICU-AW is a common complication in ICU patients and has a clinically relevant impact on short- and long-term outcomes. Several important questions remain unanswered concerning the optimal method for diagnosis and the relationship between ICU-AW and DW, delirium, muscle metabolism, and nutrition.[47] The roles of early mobilization and rehabilitation in the ICU also remain to be elucidated. Future longitudinal studies should confirm the predictive ability of early abnormalities of electrophysiological tests of peripheral nerves and muscles, muscle ultrasound imaging, and non-volitional muscle strength measurements on long-term physical dysfunction. Future efficacy nutrition trials should consider ICU-AW a clinically relevant outcome measure.[42] A multidisciplinary approach is essential and, due to the lacking of specific therapy, proper management is based on minimizing or avoiding triggers, controlling sepsis and inflammatory states, minimizing the use of sedation, strict glycemic control and early and aggressive plan of mobilization and rehabilitation to reduce muscle reconditioning and dysfunction.[48]
CIM: Critical illness myopathy

$C I P$ : Critical illness polyneuropathy

$C M A P$ : Compound muscle action potential

$E M G$ : Electromyography

$E M S$ : Electrical muscle stimulation

$G D F-15$ : Growth and differentiation factor-15

ICUAW: Intensive care unit acquired weakness

$M O F$ : Multiple organ failure

$M R C$ : Medical Research Council

NCS: Nerve conduction studies

PICS: Post intensive care syndrome

SIRS: Systemic inflammatory response syndrome

\section{References}

1. Kress JP, Hall JB. ICU-acquired weakness and recovery from critical illness. New England Journal of Medicine. 2014;370(17):1626-1635.

2. Hermans G, Van den Berghe G. Clinical review: intensive care unit acquired weakness. Critical care. 2015;19(1):274.

3. Kayambu G, Boots R, Paratz J. Physical therapy for the critically ill in the ICU: a systematic review and metaanalysis. Critical care medicine. 2013;41(6):1543-1554.

4. Batt J, dos Santos CC, Cameron JI, Herridge MS. Intensive care unit-acquired weakness: clinical phenotypes and molecular mechanisms. American journal of respiratory and critical care medicine. 2013;187(3):238-246.

5. Wollersheim T, Woehlecke J, Krebs M, Hamati J, Lodka D, Luther-Schroeder A et al., Dynamics of myosin degradation in intensive care unit-acquired weakness during severe critical illness. Intensive care medicine. 2014;40(4):528-538.

6. Latronico N, Friedrich O. Electrophysiological investigations of peripheral nerves and muscles: a method for looking at cell dysfunction in the critically ill patients.

7. Patel BK, Pohlman AS, Hall JB, Kress JP. Impact of early mobilization on glycemic control and ICUacquired weakness in critically ill patients who are mechanically ventilated. Chest. 2014;146(3):583-589.

8. Jung B, Moury PH, Mahul M, De Jong A, Galia F, Prades A et al., Diaphragmatic dysfunction in patients with ICU-acquired weakness and its impact on extubation failure. Intensive care medicine. 2016;42(5):853-861.

9. Koch S, Wollersheim T, Bierbrauer J, Haas K, Mörgeli R, Deja M, et al., Long-term recovery in critical illness myopathy is complete, contrary to polyneuropathy. Muscle \& nerve. 2014;50(3):431-436.

10. Fan E, Dowdy DW, Colantuoni E, Mendez-Tellez PA, Sevransky JE, Shanholtz C, et al., Physical complications in acute lung injury survivors: a 2-year longitudinal prospective study. Crit Care Med. 2013;42: 849-859.

11. Mirzakhani H, Williams JN, Mello J, Joseph S, Meyer MJ, Waak K, et al., Muscle weakness predicts pharyngeal dysfunction and symptomatic aspiration in long-term ventilated patients. Anesthesiology. 2013;119: 389-397. 
12. Skrzat JM. Muscle Fatigue and Recovery Across the Lifespan in Adults Who Are Healthy \& Critically Ill. Temple University; 2017.

13. Jolley SE, Bunnell AE, Hough CL. ICU-acquired weakness. Chest. 2016;150(5):1129-1140.

14. Cameron S, Ball I, Cepinskas G, Choong K, Doherty TJ, Ellis CG et al., Early mobilization in the critical care unit: A review of adult and pediatric literature. Journal of critical care. 2015;30(4):664-672.

15. Lacomis D. Electrophysiology of neuromuscular disorders in critical illness. Muscle \& nerve. 2013;47(3):452-463.

16. Friedrich OR, Van den Berghe G, Van Horebeek I, Hermans G, Rich MM, Larsson L. The sick and the weak: neuropathies/myopathies in the critically illcellular mechanisms of complex disease entities in the ICU. Physiol Rev. 2015, in press.

17. Puthucheary ZA, Rawal J, McPhail M, Connolly B, Ratnayake G, Chan P, et al., Acute skeletal muscle wasting in critical illness. JAMA. 2013;310: 1591-1600.

18. Weber-Carstens S, Schneider J, Wollersheim T, Assmann A, Bierbrauer J, Marg A, et al., Critical illness myopathy and GLUT4: significance of insulin and muscle contraction. Am J Respir Crit Care Med. 2013;187: 387-396.

19. Menorca RM, Fussell TS, Elfar JC. Nerve physiology: mechanisms of injury and recovery. Hand clinics. 2013 ;29(3):317-330.

20. Hermans G, Casaer MP, Clerckx B, Guiza F, Vanhullebusch T, Derde S, et al., Effect of tolerating macronutrient deficit on the development of intensivecare unit acquired weakness: a subanalysis of the EPaNIC trial. Lancet Respir Med. 2013;1: 621-629.

21. Demoule A, Jung B, Prodanovic H, Molinari N, Chanques G, Coirault C, et al., Diaphragm dysfunction on admission to the intensive care unit. Prevalence, risk factors, and prognostic impact - a prospective study. Am J Respir Crit Care Med. 2013;188: 213-219.

22. Skorna M, Kopacik R, Vlckova E, Adamova B, Kostalova M, Bednarik J. Small nerve fiber pathology in critical illness documented by serial skin biopsies. Muscle Nerve. 2014.

23. Wieske L, Chan Pin Yin DR, Verhamme C, Schultz MJ, van Schaik IN, Horn J. Autonomic dysfunction in ICU-acquired weakness: a prospective observational pilot study. Intensive Care Med. 2013;39: 1610-1617.

24. Kana V, Kellenberger CJ, Rushing EJ, Klein A. Muscle magnetic resonance imaging of the lower limbs: Valuable diagnostic tool in the investigation of childhood neuromuscular disorders. Neuropediatrics. 2014;45(05):278-88.

25. Foster LA, Salajegheh MK. Motor neuron disease: pathophysiology, diagnosis, and management. The American journal of medicine. 2019;132(1):32-37.

26. Godoy DA, de Mello LV, Masotti L, Di Napoli M. Intensive Care Unit Acquired Weakness (ICU-AW): a brief and practical review. Reviews in Health Care. 2015;6(1):9

27. Rhodes A, Evans LE, Alhazzani W, Levy MM, Antonelli M, Ferrer $\mathbf{R}$ et al., Surviving sepsis campaign: international guidelines for management of sepsis and septic shock: 2016. Intensive care medicine. 2017;43(3):304-377.

28. Onnolly BA, Jones GD, Curtis AA, Murphy PB, Douiri A, Hopkinson NS, et al., Clinical predictive value of manual muscle strength testing during critical illness: an observational cohort study. Crit Care. 2013;17: R229.

29. Vanpee G, Hermans G, Segers J, Gosselink R. Assessment of limb muscle strength in critically ill patients: a systematic review. Crit Care Med. 2014; 42:701-711.

30. Grimm A, Teschner U, Porzelius C, Ludewig K, Zielske J, Witte OW, et al., Muscle ultrasound for early assessment of critical illness neuromyopathy in severe sepsis. Crit Care. 2013;17: R227.

31. GiVITI Study Investigators, Latronico N, Nattino G, Guarneri B, Fagoni N, Amantini A, et al., Validation of the peroneal nerve test to diagnose critical illness polyneuropathy and myopathy in the intensive care unit: the multicentre Italian CRIMYNE-2 diagnostic accuracy study. F1000Research. 2014;3: 127.

32. Moss M, Yang M, Macht M, Sottile P, Gray L, McNulty M, et al., Screening for critical illness polyneuromyopathy with single nerve conduction studies. Intensive Care Med. 2014;40: 683-690.

33. Wieske L, Witteveen E, Petzold A, Verhamme C, Schultz MJ, van Schaik IN, et al., Neurofilaments as a plasma biomarker for ICU-acquired weakness: an observational pilot study. Crit Care. 2014;18: R18.

34. Wijdicks EF, Kramer AH. Critical care neurology part I: neurocritical care. Elsevier; 2017.

35. Brunner R, Rinner W, Haberler C, Kitzberger R, Sycha T, Herkner $\mathbf{H}$ et al., Early treatment with IgMenriched intravenous immunoglobulin does not mitigate critical illness polyneuropathy and/or myopathy in patients with multiple organ failure and SIRS/sepsis: a prospective, randomized, placebo-controlled, doubleblinded trial. Critical care. 2013;17(5): R213.

36. Zody M. Preventing Delirium through the Implementation of the ABCDE Bundle and PAD Guideline into Everyday Care in a Community Hospital Intensive Care Unit: Opportunities for Practice Improvement.

37. Elhabashy S. Cardio-Thoracic Injury, Essentials All Critical Care Nurses Need to Know. Lulu. com; 2015.

38. Blixt C. Perioperative glucose control. Inst för klinisk vetenskap, intervention och teknik/Dept of Clinical Science, Intervention and Technology; 2018.

39. Vanhorebeek I, Gunst J, Van den Berghe G. Critical care management of stress-induced hyperglycemia. Current diabetes reports. 2018;18(4):17.

40. Shepherd SJ, Newman R, Brett SJ, Griffith DM. Pharmacological therapy for the prevention and treatment of weakness after critical illness: a systematic review. Critical care medicine. 2016;44(6):1198-1205.

41. Needham DM, Wozniak AW, Hough CL, Morris PE, Dinglas VD, Jackson JC et al., Risk factors for physical impairment after acute lung injury in a national, multicenter study. American journal of respiratory and critical care medicine. 2014;189(10):1214-1224. 
42. Burke D, Gorman E, Stokes D, Lennon O. An evaluation of neuromuscular electrical stimulation in critical care using the ICF framework: a systematic review and meta- analysis. The clinical respiratory journal. 2016;10(4):407-420.

43. Fan E, Dowdy DW, Colantuoni E, Mendez-Tellez PA, Sevransky JE, Shanholtz $\mathrm{C}$ et al., Physical complications in acute lung injury survivors: a 2-year longitudinal prospective study. Critical care medicine. 2014;42(4):849.

44. Zorowitz RD. ICU-Acquired Weakness: A Rehabilitation Perspective of Diagnosis, Treatment, and Functional Management. Chest. 2016;150(4):966-971.

45. Kayambu G, Boots R, Paratz J. Early physical rehabilitation in intensive care patients with sepsis syndromes: a pilot randomised controlled trial. Intensive care medicine. 2015;41(5):865-874.

46. Ferreira NA, Lopes AJ, Ferreira AS, Ntoumenopoulos G, Dias J, Guimaraes FS. Determination of functional prognosis in hospitalized patients following an intensive care admission. World journal of critical care medicine. 2016;5(4):219.

47. Needham DM, Dinglas VD, Bienvenu OJ, Colantuoni E, Wozniak AW, Rice TW, et al., One-year outcomes in patients with acute lung injury randomised to initial trophic or full enteral feeding: prospective follow-up of EDEN randomised trial. BMJ. 2013; 346:532.

48. May MB, editor. Cardiopulmonary physiotherapy in trauma: an evidence-based approach. World Scientific; 2015. 